\title{
Epidemiología del dengue en Centroamérica y República Dominicana
}

\author{
María L. Ávila-Agüero ${ }^{1,8}$, Kattia Camacho-Badilla', José Brea Del Castillo², Lizbeth Cerezo³, \\ Lourdes Dueñas ${ }^{4}$, Marco Luque ${ }^{5}$, Mario Melgar ${ }^{6}$ y Crisanta Rocha ${ }^{7}$
}

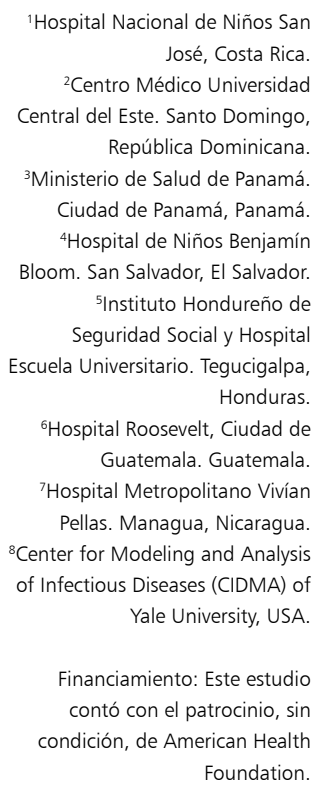

Recibido: 5 de julio de 2019 Aceptado: 10 de octubre de

\section{Introducción}

$\mathrm{E}$ 1 dengue es una enfermedad viral causada por cuatro serotipos de este virus y transmitida por mosquitos del género Aedes. Ha surgido como un grave problema de salud pública a nivel global en los últimos años, siendo la enfermedad que más ha aumentado en términos de incidencia y años vividos con enfermedad en el mundo ${ }^{1}$.

El dengue es endémico en más de 128 países $^{2,3}$ incluyendo 40 países del continente americano. Centroamérica, en particular, experimenta una alta carga de la enfermedad al reportar cerca de $8 \%$ de todos los casos del continente a pesar de sólo contar con $4 \%$ de la población continental ${ }^{4-7}$. La adecuada información epidemiológica es esencial para evaluar las estrategias de intervención en el control del dengue, incluyendo la fase de preparación ante la eventual introducción de una vacuna. En el año 2012, la Organización Mundial de la Salud (OMS) instó a los países de la Región de las Américas para que, en el año 2015 , sus sistemas de vigilancia y reporte fueran mejo$\operatorname{rados}^{4-8}$. El objetivo es conocer la carga de la enfermedad real en la región y así poder implementar estrategias con el fin de cumplir la meta de reducir para el año 2020 la morbilidad causada por dengue en $50 \%$ y la mortalidad en $25 \%$. Sin embargo, son pocas las publicaciones sobre las características epidemiológicas del dengue en la Región de las Américas y, específicamente en Centroamérica, que describan la panorámica global.

\section{Objetivos}

El objetivo principal es reportar la situación epidemiológica del dengue en Guatemala, Honduras, El Salvador, Nicaragua, Costa Rica, Panamá y República Dominicana, documentando el panorama subregional, identificando las diferencias y coincidencias en cuanto a los diferentes datos epidemiológicos recopilados. Como objetivo secundario, se pretende que este estudio sea un insumo útil para los tomadores de decisión encargados del manejo y control del dengue en nuestra región.

\section{Materiales y Métodos}

El período de estudio comprendió los años 2005 a 2014. Un médico infectólogo y un epidemiólogo de cada país (Guatemala, Honduras, El Salvador, Nicaragua, Costa Rica, Panamá y República Dominicana) recopilaron y analizaron los datos de casos y muertes por dengue de su respectivo país. Posteriormente, esta información se corroboró con los datos publicados en los boletines en línea y la base de datos interactiva de la Organización Panamericana de la Salud (OPS) además de las publica- 
ciones oficiales de los Ministerios de Salud de cada país. Se obtuvieron estadísticas poblacionales de los Institutos Nacionales de Estadística y Censo de cada país.

Se revisó el registro de los casos de dengue utilizado en cada país.

Los investigadores se reunieron presencialmente para discutir los resultados en tres ocasiones, y la epidemióloga líder (KCB) fue la encargada de analizar los datos regionales, en conjunto con la investigadora principal (MLAA).

Limitaciones de este trabajo son las propias de un estudio retrospectivo, incluyendo que los datos obtenidos no son consistentes entre los países incluidos, ya que hay diferencias en la clasificación y reporte de los casos, dificultando las comparaciones.

Este estudio no involucró la revisión de expedientes clínicos o bases de datos que contuvieran identificación de sujetos; por ende, no se violentó el derecho a la privacidad de los sujetos afectados por el dengue durante los años investigados. Los documentos revisados son públicos, de libre acceso.

\section{Resultados}

\section{Epidemiología del dengue en el nivel subregional}

\section{Incidencia}

Durante el período de estudio (2005-2014), fueron notificados 1.118.464 casos de dengue (Tabla 1). El 81,4\% de los casos fueron notificados en cuatro países: Honduras, El Salvador, Costa Rica y Nicaragua, mientras que, Guatemala, República Dominicana y Panamá contribuyeron cada uno con menos de $10 \%$ de los casos. Costa Rica, El
Salvador, Honduras y Nicaragua reportaron las tasas más altas de incidencia anual (rango 311,4-490,8 casos por cada 100.000 habitantes). Estos cuatro países tuvieron una incidencia anual de más del doble que la reportada en Guatemala, República Dominicana y Panamá (rango 80,2-114,7 por cada 100.000 habitantes).

La incidencia regional mostró un patrón trianual de un año pico, precedido y seguido por un año con menor incidencia. Los picos trianuales aumentaron sustancialmente desde el 2007. Los años 2013, 2014 y 2010 fueron los años con mayores tasas de notificación anual, superando más de seis veces la menor tasa, reportada durante el año 2008 (303-415 vs 80-90 casos por 100.000 habitantes). (Figura 1).

Tabla 1. Tasa media de incidencia y porcentaje acumulado de casos de dengue en Centro América y República Dominicana durante el período 2005-2014

\begin{tabular}{lcccc}
\hline País & $\begin{array}{c}\text { Total casos } \\
\text { dengue notificados } \\
\text { (2005-2014) }\end{array}$ & $\begin{array}{c}\text { Tasa media } \\
\text { incidencia del } \\
\text { período 2005-2014 }\end{array}$ & $\begin{array}{c}\text { Porcentaje } \\
\text { (\%) }\end{array}$ & $\begin{array}{c}\text { Porcentaje } \\
\text { acumulado } \\
\text { (\%) }\end{array}$ \\
\hline Honduras & 268.411 & 357 & 24 & 24 \\
\hline El Salvador & 238.282 & 383,6 & 21,3 & 45,3 \\
\hline Costa Rica & 220.731 & 490,8 & 19,7 & 65 \\
\hline Nicaragua & 183.252 & 311,4 & 16,4 & 81,4 \\
\hline Guatemala & 89.151 & 62,7 & 8 & 89,4 \\
\hline R. Dominicana & 78.685 & 80,2 & 7 & 96,4 \\
\hline Panamá & 39.952 & 114,7 & 3,6 & 100 \\
\hline Total & 1.118 .464 & 216,7 & 100 & \\
\hline
\end{tabular}

Fuente: Elaboración propia con base en los casos de dengue y poblaciones reportadas por las páginas oficiales de OPS y los Ministerios de Salud de los países.

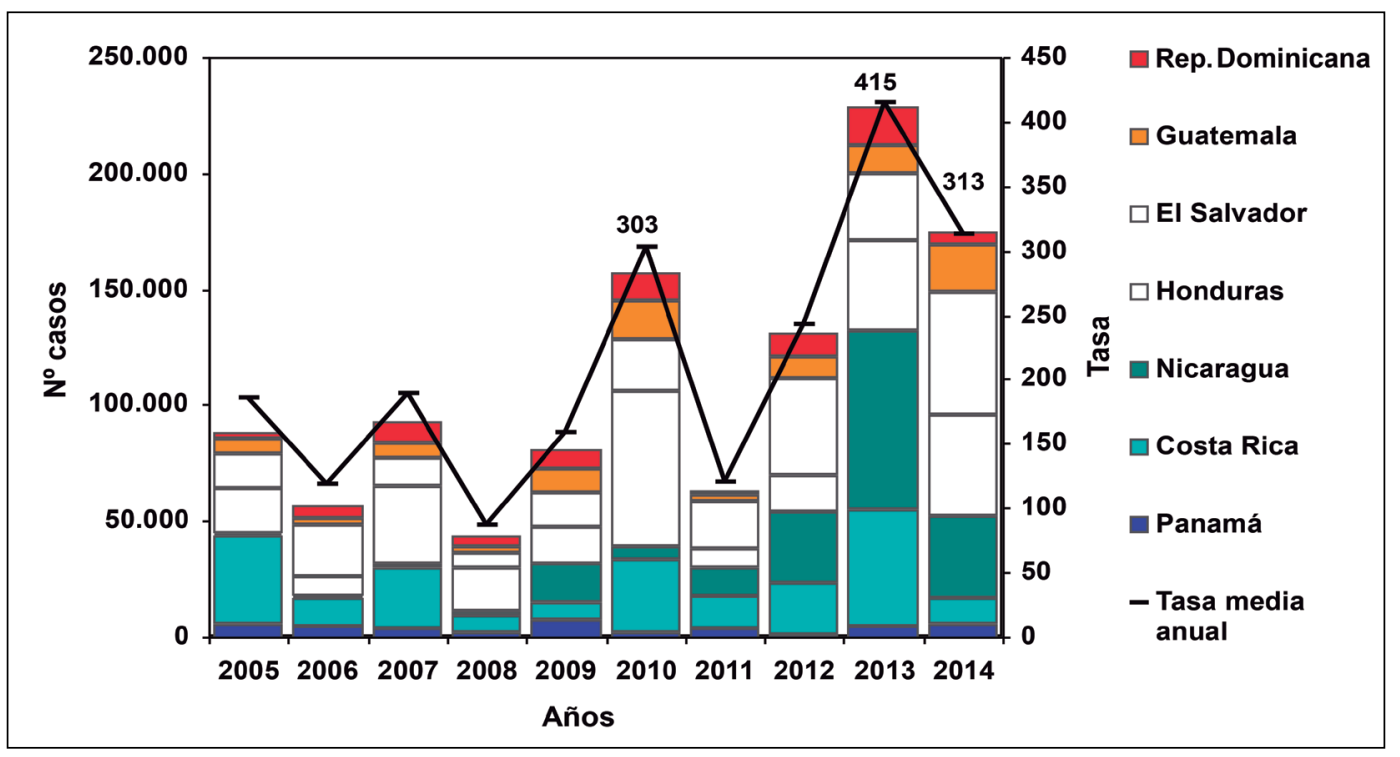

Figura 1. $N^{\circ}$ de casos notificados* $y$ tasa media anual de dengue en Centro América y República Dominicana, 2005-2014. Fuente: Elaboración propia con base en los casos reportados por las páginas oficiales de OPS/OMS y los Ministerios de Salud. *Existen diferencias en los criterios de registro de casos notificados a OPS por los países, pues, por ejemplo, Panamá notifica los casos confirmados y El Salvador notifica los casos sospechosos. 


\section{Gravedad y letalidad}

Durante el período de estudio (2005-2014) fueron reportados 32.431 casos graves, representando $2,9 \%$ de los casos notificados. Los años 2007 y 2008 fueron los dos únicos años en que se reportaron porcentajes de casos graves superiores a 5\% (Tabla 2). Honduras fue el país con la mayor cantidad y porcentaje de casos graves: 23.471 casos graves, $8,7 \%$ del total de casos notificados. Panamá reportó 120 casos graves que representan $0,30 \%$ de todos sus casos (Tabla 3 ).

En el decenio estudiado hubo 888 personas fallecidas en la región, más de la mitad de ellas ocurrieron durante los años 2009, 2010 y 2013. La letalidad por caso de dengue fue, en promedio, $0,08 \%$, con un rango anual de $0,03-0,15 \%$ y una tendencia a la baja a partir del año 2011 (Tabla 2). República Dominicana tuvo la mayor cantidad de muertes por dengue y la mayor letalidad $(0,54 \%)$, casi la misma cantidad que el resto de los países analizados. Costa Rica reportó la menor cantidad de muertes (19, eso es $2,1 \%$ de la región) y la menor letalidad $(0,01 \%)$ (Figura 2 y Tabla 3 ).

\section{Patrón de incidencia según edad y sexo}

La información acerca de la incidencia por edad sólo

Tabla 2. Incidencia, gravedad, mortalidad y letalidad por dengue a nivel subregional según año, Centro América y República Dominicana, 2005-2014

\begin{tabular}{|c|c|c|c|c|c|c|}
\hline \multirow[t]{2}{*}{ Años } & \multicolumn{3}{|c|}{ Enfermedad dengue } & \multicolumn{3}{|c|}{ Muertes por dengue } \\
\hline & Casos notificados & Casos graves & Graves/notificados (\%) & $\mathbf{n}$ & $\%$ & Letalidad (\% casos) \\
\hline 2005 & 88.568 & 2.349 & 2,7 & 45 & 5,1 & 0,05 \\
\hline 2007 & 93.082 & 4.998 & 5,4 & 86 & 9,7 & 0,09 \\
\hline 2008 & 43.791 & 2.756 & 6,3 & 49 & 5,5 & 0,11 \\
\hline 2011 & 63.652 & 1.293 & 2 & 37 & 4,2 & 0,06 \\
\hline 2012 & 130.630 & 3.799 & 2,9 & 72 & 8,1 & 0,06 \\
\hline 2013 & 228.494 & 5.594 & 2,4 & 174 & 19,6 & 0,08 \\
\hline 2014 & 175.067 & 3.363 & 1,9 & 60 & 6,8 & 0,03 \\
\hline
\end{tabular}

Fuente: Elaboración propia con base en los casos de dengue según gravedad y defunciones reportadas en las páginas oficiales de OPS y los Ministerios de Salud de los países.

\begin{tabular}{|c|c|c|c|c|c|c|}
\hline \multirow[t]{2}{*}{ País } & \multicolumn{3}{|c|}{ Enfermedad dengue } & \multicolumn{3}{|c|}{ Muertes por dengue } \\
\hline & Casos notificados & Casos graves & Graves/notificados (\%) & $\mathbf{n}$ & $\%$ & Letalidad ( $\%$ casos) \\
\hline Guatemala & 89.151 & 704 & 0,8 & 122 & 13,7 & 0,14 \\
\hline Honduras & 268.411 & 23.471 & 8,7 & 165 & 18,6 & 0,06 \\
\hline El Salvador & 238.282 & 2.730 & 1,1 & 44 & 5 & 0,02 \\
\hline Nicaragua & 183.252 & 805 & 0,4 & 70 & 7,9 & 0,04 \\
\hline Costa Rica & 220.731 & 760 & 0,3 & 19 & 2,1 & 0,01 \\
\hline Panamá & 39.952 & 120 & 0,3 & 43 & 4,8 & 0,11 \\
\hline R. Dominicana & 78.685 & 3.841 & 4,9 & 425 & 47,9 & 0,54 \\
\hline Total & 1.118 .464 & 32.431 & 2,9 & 888 & 100 & 0,08 \\
\hline
\end{tabular}


estaba disponible para Honduras, El Salvador, Panamá y República Dominicana. En ninguno de estos países hubo diferencias significativas en la incidencia por sexo $(\mathrm{p}<$ 0,85 ). Identificamos una diferencia en la carga por grupo etario en Panamá donde la mayor incidencia ocurrió en mayores de 25 años $(62,8 \%)$ con respecto a la de Honduras, El Salvador y República Dominicana, donde la mayoría de las notificaciones se presentó en pacientes bajo 14 años de edad (62,3\%, 72,3\% y 64\%, respectivamente) (Figura 3).

\section{Circulación de serotipos}

Los cuatro serotipos de dengue circularon en la subregión durante la década analizada (Figura 4). Guatemala, Honduras, El Salvador y Nicaragua reportaron circulación de los cuatro serotipos, mientras que en Costa Rica y Panamá no hubo certeza de la circulación del DENV-4 y en República Dominicana no se reportó la circulación del DENV-3.

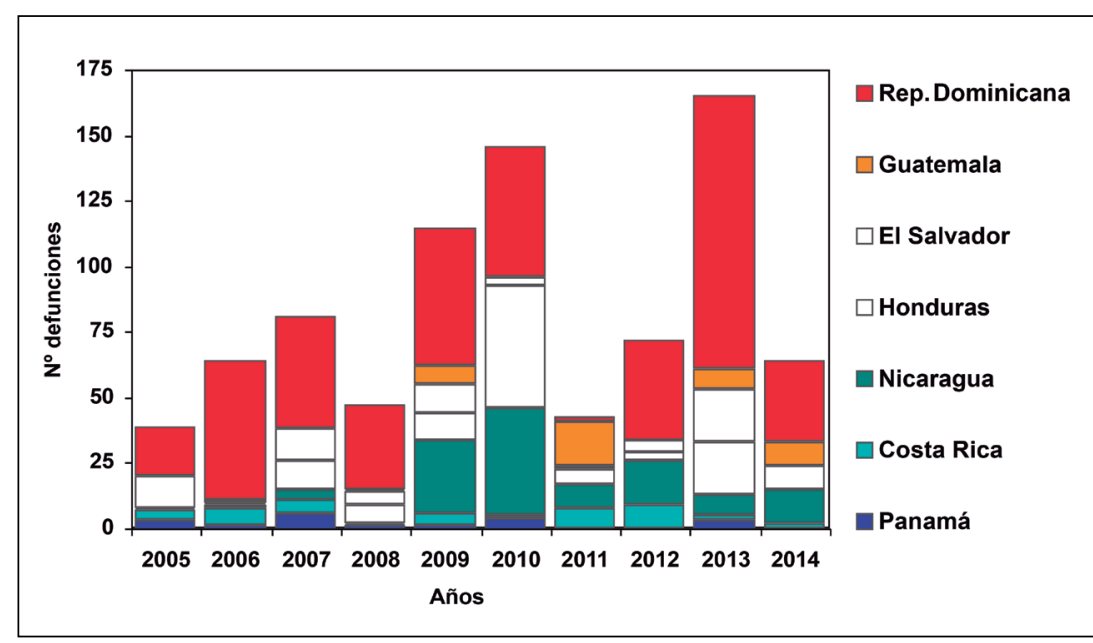

Figura 2. № de defunciones por dengue reportadas según año en Centro América y República Dominicana, 2005-2014. Fuente: Elaboración propia con base en los casos reportados por las páginas oficiales de los Ministerios de Salud de los países.

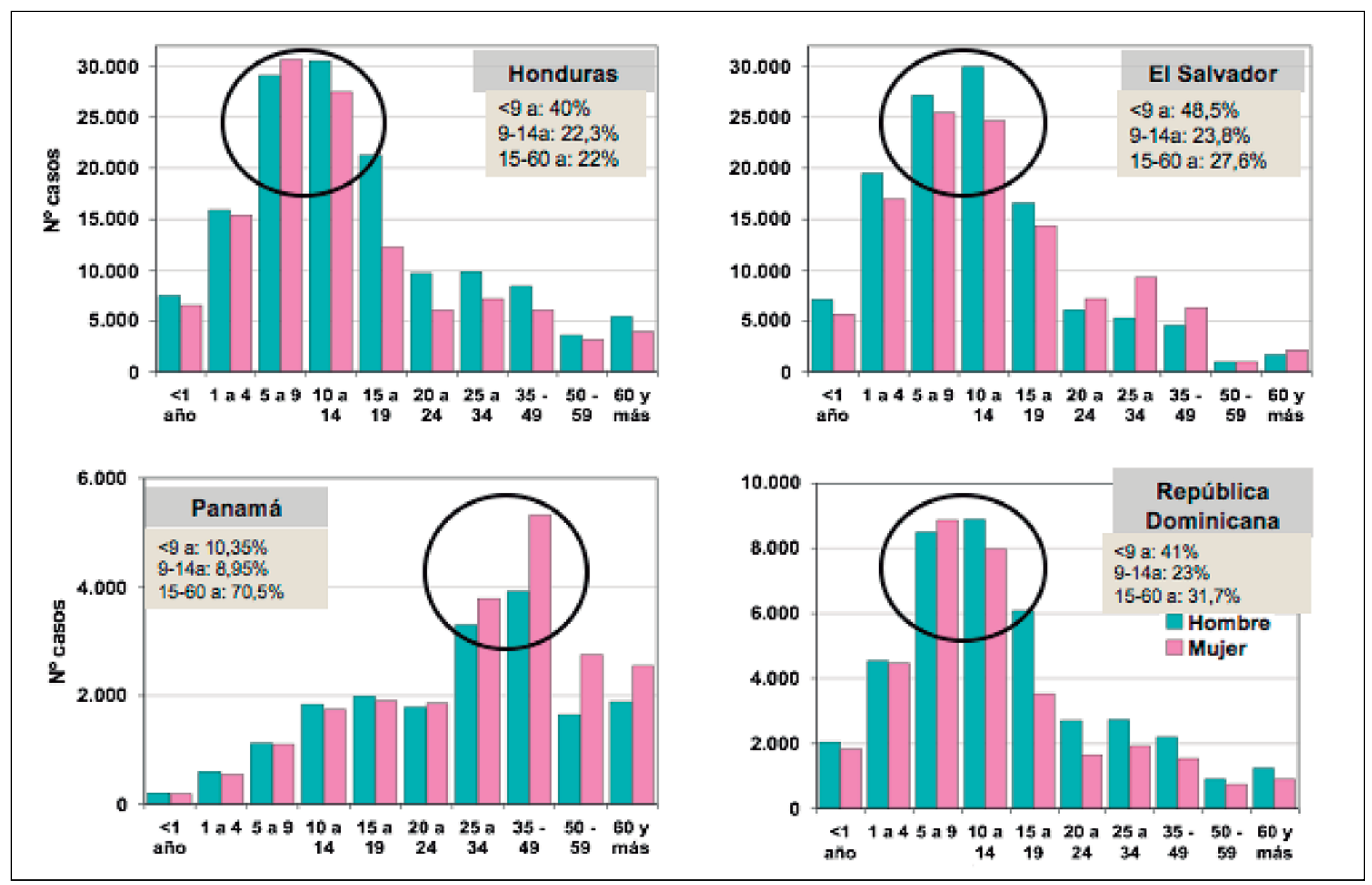

Figura 3. No de casos notificados de dengue según grupo de edad y sexo en países de Centro América y República Dominicana, 2005 - 2014 . Fuente: Elaboración propia con base en los datos reportados por las páginas oficiales de los Ministerios de Salud de los países. 
Figura 4. Serotipos circulantes en Centro América y República Dominicana, 20052014. Fuente: Elaboración propia con base en los datos reportados por las páginas oficiales de los Ministerios de Salud de los países.

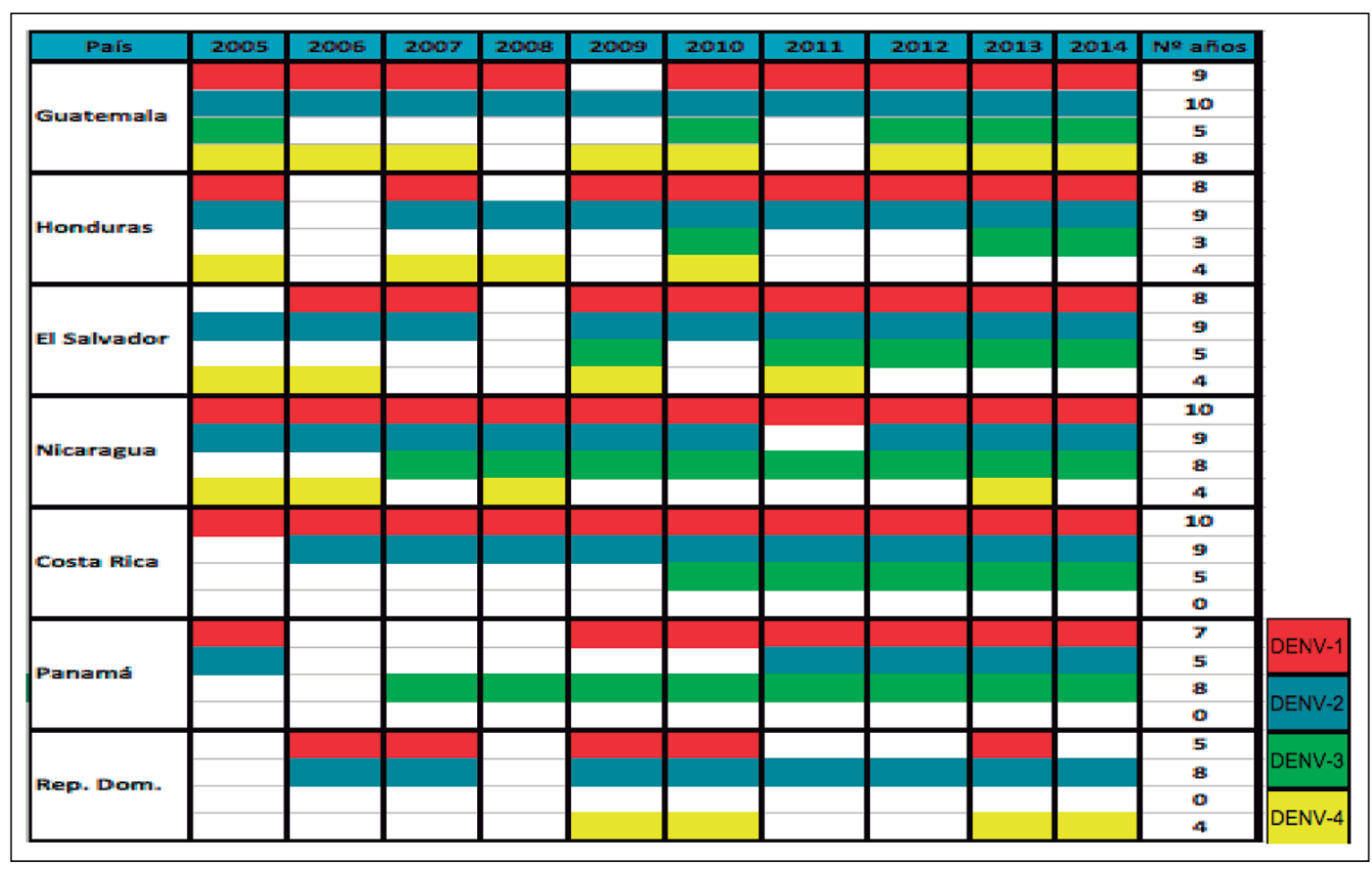

\section{Discusión}

A pesar que el dengue es una condición endémica en Centro América, con graves repercusiones para la salud y la economía de los países, no hay hasta la fecha, una publicación que reúna las características epidemiológicas de la infección en la región.

Esta investigación es un primer intento que permite constatar cómo una región relativamente pequeña tiene gran variabilidad en la presentación epidemiológica del dengue careciendo de un proceso similar de reporte. Ello afecta la uniformidad de los criterios de reporte epidemiológico y las definiciones de caso, incluyendo las muertes por dengue. La calidad del dato no permite interpretar con mayor rigurosidad, las diferencias en las tasas de incidencia, la proporción de casos graves y la tasa de letalidad entre países. El acceso a la información fue complejo. Los datos de cada país difieren según sea la calidad del registro y la forma en cómo se realiza el envío de reportes, y de los sistemas de información para la captura, tabulación y análisis de los datos. La información recolectada presentaba inconsistencias entre las variables, o al compararlos con otras fuentes de datos; mientras que, según el país, hay datos que no son recolectados como parte de la vigilancia de dengue. Desde 2009, los criterios actualizados de la OMS/OPS para el diagnóstico de dengue probable, dengue confirmado por laboratorio, dengue con o sin signos de alarma y dengue grave, se han incorporado progresivamente a los programas de vigilancia de los países de la región. Sin duda, un paso necesario será uniformar criterios de reporte, para poder contar con datos más comparables y tener cargas de la enfermedad más cercanas a la realidad en nuestra subregión.

Es conocida la tendencia de los sistemas pasivos de vigilancia nacional a notificar menos casos de dengue. Las estimaciones del número de casos de dengue informados por los sistemas nacionales de vigilancia en América Latina pueden ser hasta 28 veces menores que el número de casos reales ${ }^{9-14}$, reportándose menos casos en adultos que en niños ${ }^{12}$. Por lo que la verdadera carga del dengue es subestimada con tasas de incidencia reales que podrían ser de 10 a 20 veces mayores de las que se informan en algunos países ${ }^{9-16}$.

La vigilancia de la enfermedad del dengue ha sido obstaculizada por las grandes diferencias entre los casos informados y estimados debido a la calidad variable de los datos disponibles ${ }^{17-19}$. Hallazgos similares a los nuestros los reportan Torres JR y cols. ${ }^{20}$, mencionando las lagunas significativas entre los datos de vigilancia notificados a la OPS y los que ellos identificaron en los estudios publicados, y concluyen que, en general, se notificaron menos casos confirmados y muertes a la OPS que en los estudios publicados.

Por ende, es claro que existe un subregistro importante de los casos de dengue en nuestra subregión, ya que, de 
contar con datos de mayor calidad y de reporte uniforme, la incidencia sería exponencialmente mayor.

El reporte de los serotipos circulantes depende del número de muestras que se recolectan en las áreas donde circula el virus y de la oportunidad de la toma de la muestra. Cada país cuenta con distintas técnicas de diagnóstico de la enfermedad y, por lo general, no se realizan pruebas diagnósticas a todos los casos notificados como sospechosos ${ }^{16}$. Sin embargo, nuestros datos evidencian la circulación de los cuatro serotipos en la subregión, lo que implica un alto nivel de hiperendemicidad y por ende, un mayor riesgo de casos graves. Esta presunción parece cumplirse durante el 2019, ya que Honduras, Nicaragua y Guatemala han declarado alerta epidemiológica con aumento en los casos, y en Honduras con alta letalidad ${ }^{19}$.

Durante el período 2005-2014, el dengue en Centroamérica y en República Dominicana tuvo un comportamiento cíclico; endémico durante todo el período, e incidencias mayores en los años 2010, 2013 y 2014, lo que puede estar relacionado con el hecho que en el año 2009 se publicó la actualización de las guías para el manejo del dengue, por parte de la $\mathrm{OMS}^{17}$, como previamente fue comentado. Desde la década de 1980, la incidencia del dengue se ha multiplicado por 30 , cifras observables en esta publicación. Sin embargo, en el año 2017, hubo una reducción notable en los casos notificados de incidencia de dengue en las Américas, incluidos los casos graves y fatales. Es posible que eventos multifactoriales expliquen la disminución del dengue observada en 2017, entre ellos, el agotamiento de susceptibles, mayor control de vectores, e incluso, cambios o adaptaciones de vectores y virus. Ello evidencia lo cambiante que es la epidemiología del dengue y la necesidad de actualizar los conocimientos sobre su comportamiento.

Se identificaron notorias diferencias entre los países, sin un patrón epidemiológico homogéneo. En Honduras, El Salvador y República Dominicana las mayores tasas de incidencia y hospitalizaciones se han dado en edades menores de 19 años, mientras que en Panamá ha sido en la población entre 25 y 49 años. La gravedad reportada de los casos fue mayor en los años 2007 y 2008 (5,4 y 6,3\% de casos graves, respectivamente), manteniendo porcentajes entre 2 y $3 \%$ durante los últimos años. República Dominicana es el país con mayor letalidad, con una cifra promedio del período que supera siete veces el promedio subregional y Costa Rica es el de menor letalidad. Sin embargo, los dominicanos reportan muertes por dengue que no necesariamente tienen confirmación por laboratorio. En El Salvador, República Dominicana, Honduras y Panamá, la letalidad afecta principalmente a los niños bajo 1 año de edad y a los adultos sobre 55 años de edad.

La mortalidad por dengue en la región varía de acuerdo a cada país. Costa Rica registra $2,1 \%$ de las muertes y República Dominicana 50,8\% de las defunciones de todo el período. Estas diferencias porcentuales ameritan una investigación más a fondo para poder identificar cuáles son las variables que las determinan. El acceso oportuno a los servicios de salud, el porcentaje de cobertura universal de seguros de salud, el diagnóstico y el manejo temprano de los casos y sus complicaciones, siguiendo protocolos estrictos, deben ser los objetivos primordiales para lograr reducir la mortalidad y la letalidad.

Las diferencias en el nivel de acceso a los servicios de salud y la calidad del diagnóstico clínico y por laboratorio, deben considerarse al interpretar las tasas de incidencia y mortalidad por dengue entre países, ya que son parte de que explica las notorias diferencias.

Costa Rica tiene un sistema de salud universal dado por un único prestador público de atención de la enfermedad, la Caja Costarricense del Seguro Social con una cobertura contributiva de $95 \%$ para el año $2016^{15}$, y el Ministerio de Salud que ejecuta funciones de rectoría. En el resto de los países hay dos o más proveedores públicos de atención a la enfermedad, entre ellos los mismos Ministerios de Salud. En República Dominicana, por ejemplo, la cobertura de seguro en salud ha tenido un incremento importante en los últimos años, pasando de $43 \%$ en el 2011 a $65 \%$ en el 2015, aunque persiste una brecha importante para una parte significativa de la población. En Honduras, 9 de cada 10 personas no están cubiertas por seguro de salud alguno y se estima que cerca de $18 \%$ de la población (más de 1,5 millones de hondureños) no tiene acceso a los servicios de salud (Instituto de Acceso a la Información Pública [2015] Portal único de Transparencia. Secretaria de Salud).

Dmytraczenko y Almeida ${ }^{21}$, a pesar de reconocer los avances de la región en cuanto a cobertura de salud universal, ahondan en estas diferencias, y mencionan que las brechas entre los ricos y los pobres son más amplias para la atención en los hospitales. Lo cual, puede influir en las diferentes tasas de mortalidad que se reportan en los países centroamericanos.

La complejidad del dengue, del control vectorial y de la implementación de la estrategia de gestión integrada (EGI), requiere un nuevo elemento. La esperanza sigue cifrada en una vacuna segura y eficaz que permita reducir la morbi-mortalidad y lograr los objetivos que la OMS ha propuesto. Luego de un largo proceso, la primera vacuna CYD-TDV (Dengvaxia) de Sanofi-Pasteur- contra el dengue ha sido aprobada por la OMS, para ser aplicada en áreas de alta transmisión y alta endemicidad ${ }^{22-24}$. Sin embargo, a pesar que se considera una vacuna segura y eficaz, recientes datos ${ }^{25}$ indujeron al proveedor y a la OMS a solicitar que previo a la aplicación de la vacuna se realicen estudios de laboratorio que indiquen que el paciente es seropositivo para dengue, aunque el riesgo de desarrollar dengue grave en sujetos vacunados, de previos seronegativos, cuando se exponen al virus salvaje circulante, es bajo. Este aspecto atrasa la utilización de 
la vacuna como una herramienta más de control, y es posible que retrase la aprobación de otras vacunas hasta tanto no demuestren que no ocurre lo mismo que con CYD-TDV. Por otro lado, no existe la "prueba ideal" que de manera rápida y confiable demuestre la exposición previa, sobre todo por los falsos positivos que puede dar una exposición previa a zika, chikungunya $u$ otras arbovirosis ${ }^{26-27}$. Además, surge la pregunta de cada cuánto tiempo se deberá hacer el tamizaje individual, y si las encuestas poblacionales de seroprevalencia son lo suficientemente confiables, lo que ha sido discutido en el documento elaborado por la Sociedad Latinoamericana de Infectología Pediátrica (SLIPE) ${ }^{28}$.

El SAGE (Grupo de Expertos en Asesoramiento Estratégico sobre Inmunización) de la OMS en la reunión de abril de $2018^{29}$ concluyó que para los países que consideren la vacunación con CYD-TDV como parte de su programa de control del dengue, una estrategia de detección previa a la vacunación, en la que sólo las personas seropositivas al dengue serán vacunadas, es la opción preferida. Lo ideal sería utilizar una prueba con la mayor especificidad para minimizar el uso involuntario de la vacuna en personas seronegativas. Se podrían considerar dos tipos de pruebas: las pruebas serológicas (IgG ELISA) o las pruebas de diagnóstico rápido (PDR). Los ensayos ELISA no proporcionan información sobre el punto de cohorte para el estado serológico de un individuo, y los PDR disponibles actualmente, no se han validado aún con el propósito de detectar una infección previa por dengue. Sin embargo, cualquiera de las dos opciones podría considerarse en entornos de alta prevalencia hasta que haya mejores pruebas disponibles.

En nuestra subregión no hay estudios de seroprevalencia recientes; Nicaragua ${ }^{30}$, reporta en una población de 999 niños entre los 4 y 16 años, una seroprevalencia global $91 \%$ (75\% a los 4 años, $100 \%$ a los 16 años). En Costa Rica se investigaron 206 niños, demostrando en la zona costera $36,9 \%$ de seroprevalencia vs $2,9 \%$ en el área montañosa ${ }^{31}$.

Infiriendo de estos datos, se puede decir que las actuales seroprevalencias han de ser más altas, para más grupos poblacionales en Centroamérica, sobre todo tomando en consideración las incidencias reportadas en este estudio.

En tanto, la información clínica y epidemiológica indica que la gran mayoría de los países de la región tienen tasas de incidencia alta, que han fluctuado en los últimos años, con co-circulación significativa de varios serotipos a la vez y con costos cada vez mayores en la atención médica de los casos de dengue y en el control del vector.

\section{Conclusiones}

La introducción del virus de chikungunya en el año 2013 y del virus de zika en el 2015, ponen de manifiesto la vulnerabilidad regional a las arbovirosis transmitidas por Aedes sp y la limitada capacidad de respuesta de los sistemas de salud.

Con los datos presentados, es muy evidente que el dengue es una enfermedad vectorial endémica en nuestros países, que representa una carga económica muy elevada para los sistemas de salud y que, a pesar de las medidas de salud pública que se han implementado como parte de las estrategias del control del dengue, no se ha podido eliminar esta enfermedad, produciendo una significativa morbilidad y mortalidad. La vacuna contra el dengue es una necesidad, deberá ser el complemento a la EGI contra el dengue. En el año 2017 y principios de 2018 estábamos en un "silencio epidemiológico" que mantuvo a la infección por dengue estable; sin embargo, ello podía implicar que se estaban acumulando susceptibles que generarían en algunos años brotes importantes, y esto es al parecer lo que está ocurriendo en el 2019.

Una vez revisada la epidemiología del dengue en la subregión de Centroamérica y República Dominicana, estudiada la carga de enfermedad durante un período de 10 años, analizados los estudios de costo-efectividad de la vacuna y revisados los estudios de eficacia presentados por la vacuna aprobada hasta el momento, diferentes escenarios deberán ser considerados para elaborar y ejecutar las mejores estrategias de control.

No cabe duda que el dengue es un problema de Salud Pública $^{32-35}$ a nivel mundial que representa un alto impacto económico y social para los Sistemas de Salud, y que para Centroamérica y República Dominicana no es la excepción.

El reloj del dengue marca tic-tac, pero no da la hora.

Agradecimiento: A Ana Morice-Trejos por la revisión critica del documento y su aporte en la elaboración de los gráficos y figuras.

\section{Resumen}

Introducción: Centroamérica experimenta una alta carga de la enfermedad por dengue aportando cerca de $8 \%$ de todos los casos del continente. Este trabajo reporta la epidemiología del dengue en la subregión en un período de 10 años. Objetivos: Documentar la epidemiología del dengue en Centro América y República Dominicana. Material y Métodos: Período de estudio: años 2005-2014. Se recopilaron y analizaron los datos de casos y muertes por dengue de los países de Centro América y República Dominicana, reportados por los Ministerios de Salud y se corroboró con los datos publicados en los boletines en línea y la base de datos interactiva de la Organización Pa- 
namericana de la Salud (OPS). Se obtuvieron estadísticas poblacionales de los Institutos Nacionales de Estadística y Censo de cada país. Resultados: Durante el período de estudio fueron notificados 1.118 .464 casos de dengue. Reportados 32.431 casos graves, 888 personas fallecidas. La letalidad por caso de dengue fue en promedio $0,08 \%$. Los cuatro serotipos de dengue circularon durante el decenio estudiado. Discusión: La información clínica y epidemiológica, indica tasas de incidencia alta, que han fluctuado en los últimos años, con co-circulación significativa de varios serotipos a la vez. Conclusiones: Se identificaron diferencias notorias en la recolección de datos de la vigilancia entre países. Se determinó un patrón epidemiológico heterogéneo.

\section{Referencias bibliográficas}

1.- Vos T, Barber R M, Bell B, et al. Global, regional, and national incidence, prevalence, and years lived with disability for 301 acute and chronic diseases and injuries in 188 countries, 1990-2013: a systematic analysis for the Global Burden of Disease Study 2013. Lancet 2015; 386 (9995): 743-800. doi: 10.1016/S01406736(15)60692-4.

2.- Bhatt S, Gething P W, Brady O J, Messina J P, Farlow A W, Moyes C L, et al. The global distribution and burden of dengue. Nature. 2013; 496 (7446): 504-7 doi: 10.1038/ nature 12060 .

3.- Brady O J, Gething P W, Bhatt S, Messina J P, Brownstein J S, Hoen A G, et al. Refining the global spatial limits of dengue virus transmission by evidence-based consensus. PLoS Negl Trop Dis 2012; 6 (8): e1760. doi: 10.1371/journal.pntd.0001760.

4.- Organización Panamericana de la Salud: Definiciones de casos. Dengue. Boletín Epidemiológico 2000; 21 (2): 14-15. http://cidbimena.desastres.hn/docum/ops/ publicaciones/Epidemiologico/be_v21n2.pdf.

5.- Pan American Health Organization: Dengue and dengue hemorrhagic fever in the Americas: Guidelines for Prevention and Control. Scientific Publication No. 548, Washington, 1994, 98 pp. http://iris.paho.org/xmlui/ handle/123456789/40300.

6.- Organización Panamericana de la Salud: El dengue y la fiebre hemorrágica de dengue en las Américas: una visión general del problema. Boletín Epidemiológico 1992; 13 (1): 9-10. http://iris.paho.org/xmlui/ bitstream/handle/123456789/37997/14448. pdf? sequence $=1 \&$ is Allowed $=y$.

7.- Organización Panamericana de la Salud: Resurgimiento del dengue en las Américas. Boletín Epidemiológico 1997; 18 (2): 1-6. http://iris.paho.org/xmlui/ handle/123456789/46257.

8.- Organización Panamericana de la Salud: Dengue y fiebre hemorrágica del dengue, 1996. Boletín Epidemiológico 1996; 17 (4): 12-14. http://iris.paho.org/xmlui/bitstream/ handle/123456789/46272/BE_v17n4. pdf? sequence $=1 \&$ isAllowed $=\bar{y}$.

9.- $\quad$ Shepard D, Undurraga E, Halasa Y, Stanaway
J. The global economic burden of dengue: a systematic analysis. Lancet Infect Dis 2016; 16 (8): 935-41. doi: 10.1016/S14733099(16)00146-8.

10.- Shepard D, Coudeville L, Halasa Y, Zambrano B, Dayan, G. Economic impact of dengue illness in the Americas. Am J Trop Med Hyg 2011; 84 (2): 200-7. doi: 10.4269/ ajtmh.2011.10-0503.

11.- WHO. Wkly Epidemiol Rec - July 2016 [Internet]. who.int. 2016 [cited 18 August 2016]. Available from: http://www.who.int/ wer/2016/wer9130/en/

12.- Sarti E, L'Azou M, Mercado M, Kuri P, Siqueira J, Solís E, et al. A comparative study on active and passive epidemiological surveillance for dengue in five countries of Latin America. Int J Infect Dis 2016; 44: 44-9. doi:10.1016/j.ijid.2016.01.015.

13.- WHO PAHO. Number of reported cases of dengue and severe dengue (SD) in the Americas, by country: Figures for 2015 (to week noted by each country) [Internet]. Paho.org. 2016 [cited 19 August 2016]. Available from: http:/www.paho.org/hq/ index.php?option $=$ com_docman $\&$ task $=$ doc view\&Itemid=270\&gid=32910\&1 ang=en.

14.- WHO Global strategy for dengue prevention and control 2012-2020. https://www.who.int/ denguecontrol/9789241504034/en/

15.- Caja Costarricense del Seguro Social Área de Estadística en Salud 2017. https://www.ccss. sa.cr/est_salud.

16.- Standish K, Kuan G, Avilés W, Balmaseda A, Harris E. High dengue case capture rate in four years of a cohort study in Nicaragua compared to national surveillance data PLoS Negl Trop Dis 2010; 16;4(3):e633 doi: 10.1371/journal. pntd.0000633.

17.- Toan N T, Rossi S, Prisco G, Nante N, Viviani $\mathrm{S}$. Dengue epidemiology in selected endemic countries: factors influencing expansion factors as estimates of underreporting Trop Med Int Health 2015; 20 (7): 840-63 doi: 10.1111/ tmi. 12498 .

18.- Dengue guidelines for diagnosis, treatment, prevention and control. TDR [Internet]. 2009 [cited 22 August 2016]. Available from: http://www.who.int/tdr/publications/trainingguideline-publications/ dengue-diagnosistreatment/en/
19.- Organización Panamericana de la Salud/ Organización Mundial de la Salud. Actualización epidemiológica: dengue. 9 de agosto de 2019, Washington, D.C. OPS/OMS. 2019. https://www. paho.org/hq/index.php?option $=\mathrm{com}_{-}$ docman\&view = download\&category slug=dengue-2158\&alias $=49621-9$-de-agostode-2019-dengue-actualizacion-epidemiologica1\&Itemid=270\&lang=es.

20.- Torres J R, Orduna T A, Piña-Pozas M, Vázquez-Vega D, Sarti E. Epidemiological characteristics of dengue disease in Latin America and in the Caribbean: A systematic review of the literature J Trop Med. 2017; 2017: ID 8045435. doi: 10.1155/2017/8045435.

21.- Dmytraczenko T, Almeida G, eds. 2015. Toward universal health coverage and equity in Latin America and the Caribbean: evidence from selected countries. Directions in development. Washington, DC: World Bank Group. https://www.paho.org/hq/ dmdocuments/2015/UHC-PAHO-WB-2015.pdf

22.- Capeding M R, Tran N H, Hadinegoro S R, Ismail H I, Chotpitayasunondh T, Chua M N, et al. Clinical efficacy and safety of a novel tetravalent dengue vaccine in healthy children in Asia: a phase 3, randomised, observermasked, placebo-controlled trial. Lancet 2014; 384 (9951): 1358-65. doi: 10.1016/S01406736(14)61060-6.

23.- Hadinegoro S R, Arredondo-García J L, Capeding M R, Deseda C, Chotpitayasunondh T, Dietze R, et al. Efficacy and long-term safety of a dengue vaccine in regions of endemic disease. N Engl J Med 2015; 373 (13): 1195206. doi: 10.1056/NEJMoa1506223.

24.- Gessner B D, Wilder-Smith A. Estimating the public health importance of the CYDtetravalent dengue vaccine: Vaccine preventable disease incidence and numbers needed to vaccinate. Vaccine $2016 ; 29 ; 34$ (20): 2397-401. doi: 10.1016/j. vaccine.2016.03.017.

25.- Sridhar S, Luedtke A, Langevin E, Zhu M, Bonaparte M, Machabert T, et al. Effect of dengue serostatus on dengue vaccine safety and efficacy. N Engl J Med 2018; 26; 379 (4): 32740. doi: 10.1056/NEJMoa1800820.

26.- Cassadou S, Boucau S, Petit-Sinturel M, Huc P, Leparc-Goffart I, Ledrans M. Emergence 
of chikungunya fever on the French side of Saint Martin island, October to December 2013. Euro Surveill. 2014; 19 (13): pii=20752. doi: 10.2807/1560-7917.es2014.19.13.20752.

27.- WHO 2016. WHO statement on the first meeting of the International Health Regulations (2005) (IHR 2005) Emergency Committee on Zika virus and observed increase in neurological disorders and neonatal malformations http://www.who. $\mathrm{int} /$ mediacentre/news/statements/2016/1stemergency-committee-zika/en/

28.- SLIPE Documento de posición: vacunas contra el dengue. http://www.slipe.org/noticias_ver. asp?idNoticia $=141$.

29.- WHO Weekly epidemiological record 2018; 23 (93): 329-44. https://apps.who.int/iris/bitstream/ handle/10665/272782/WER9323.pdf?ua=1.
30.- Balmaseda A, Hammond S N, Tellez Y, Imhoff L, Rodríguez Y, Saborío SI, et al. High seroprevalence of antibodies against dengue virus in a prospective study of schoolchildren in Managua, Nicaragua Trop Med Int Health 2006; 11 (6): 935-4. doi: 10.1111/j.13653156.2006.01641.x.

31.- Iturrino-Monge R, Ávila-Aguero M L, ÁvilaAguero C R, Moya-Moya T, Cañas-Coto A, Camacho-Badilla K, et al. Seroprevalence of dengue virus antibodies in asymptomatic Costa Rican children, 2002-2003: A pilot study. Rev Panam Salud Publica, 2006; 20 (1): 39-43. doi: 10.1590/s1020-49892006000700005.

32.- Meltzer M I, Rigau-Pérez J G, Clark G G, Reiter P, Gubler D J. Using disability-adjusted life years to assess the economic impact of dengue in Puerto Rico: 1984-1994. Am J Trop
Med Hyg, 1998; 59(2): 265-71 doi: 10.4269/ ajtmh.1998.59.265

33.- Shepard D S, Undurraga E A, BetancourtCravioto M, Guzmán M G, Halstead S B, Harris E, et al. Approaches to refining estimates of global burden and economics of dengue. PLoS Negl Trop Dis 2014; 20; 8 (11): e3306 doi: 10.1371/journal.pntd.0003306.

34.- San Martín J L, Brathwaite O, Zambrano B, Solórzano J O, Bouckenooghe A, Gustavo H, et al. The epidemiology of dengue in the Americas over the last three decades: a worrisome reality. Am J Trop Med Hyg, 2010; 82 (1): 128-35. doi: 10.4269/ajtmh.2010.09-0346.

35.- Zambrano B, San Martín J L. Epidemiology of dengue in Latin America. J Pediatric Infect Dis Soc 2014; 3 (3): 181-2. doi: 10.1093/jpids/ piu071. 